\title{
Familial Multiple Myeloma: Report on Two Families and Discussion of Screening Options
}

\author{
Erica H. Gerkes', Mirjam M. de Jong', Rolf H. Sijmons', Edo Vellenga² \\ 1 Department of Genetics, University Medical Centre Groningen, University of Groningen, Groningen, the Netherlands \\ 2 Department of Haematology, University Medical Centre Groningen, University of Groningen, Groningen, the Netherlands
}

Key words: multiple myeloma, familial, screening

Corresponding author: Erica H. Gerkes, M.D., Department of Genetics, University Medical Centre Groningen, Hanzeplein 1, P0 Box 30.001, 9700 RB Groningen, the Netherlands, tel.: +31 50 3617235, e-mail: E.H.Gerkes@medgen.umcg.nl

Submitted: 15 May 2007

Accepted: 4 June 2007

\begin{abstract}
Multiple myeloma (MM) is a relatively rare haematological malignancy seen in older persons. It has an unknown aetiology and usually occurs incidentally within a family. However, several families have been reported with multiple cases of MM, so that the existence of hereditary MM has been postulated although no causative germline mutations have been detected so far. First-degree relatives of MM patients have been reported to have a relative risk between two and four times higher than normal of developing $M M$ and we presume the risks are higher for relatives in the case of familial MM. Here we report on two families with MM who requested presymptomatic screening of healthy relatives. Although risk estimates for asymptomatic relatives in these types of families are not available, a clinically significant risk of developing MM cannot be excluded. We suggest that, in a research setting, screening for MM could be offered to individuals with more than one first-degree affected relative, or to those with one first-degree and at least one second-degree relative with $M M$. We propose a screening programme of annual protein electrophoresis of blood and urine, starting at age 40 (or earlier if a family member presented with MM at a younger age).
\end{abstract}

\section{Introduction}

Multiple myeloma (MM) is a haematological malignancy characterised by a malignant proliferation of monoclonal plasma cells in the bone marrow producing monoclonal immunoglobulins and by the formation of focal osteolytic lesions in the skeleton. The disorder might evolve from a common pre-malignant condition called monoclonal gammopathy of undetermined significance (MGUS). However, the factors driving the malignant transformation of MGUS are as yet unknown. The clinical manifestations of MM include bone pains caused by lytic bone lesions, anaemia, hypercalcaemia, immunodeficiency and renal failure [1,2]. The incidence of the disease is about 3 to 4 per 100,000 in the Netherlands and mortality rates are only slightly lower [3]. Although the disorder is not curable in most cases, the overall survival varies depending on the age of onset and other prognostic features. The five-year survival rate is approximately $30 \%$ $[1,3,4]$.

MM usually occurs incidentally within a family. However, several families have been described with multiple cases of MM, suggesting there may be a genetic predisposition [5-11]. Since no causative germline gene mutations have been identified, diagnostic DNA testing of families with an inherited type of MM and presymptomatic genetic screening for unaffected relatives are unavailable. 
Here we report on two families with familial clustering of $M M$, we review the literature on familial MM, and we discuss the options for screening healthy relatives for $M M$ in these familial cases.

\section{Case reports}

Two unrelated families were referred to our clinic asking about the possible heritability of $M M$ in their family and the options for screening of healthy relatives. The patients' medical records were reviewed.

Family 1 had three first-degree relatives affected by $M M$ (Figure 1). The index patient (III-2) was a 65-year old female who was diagnosed with MM after presenting with pain in the shoulder region and fatigue. She had an lgG paraproteinaemia of $32 \mathrm{~g} / \mathrm{l}$, which later rose to $53 \mathrm{~g} / \mathrm{l}$, and 65 percent bone marrow plasma cells. Cytogenetic findings in bone marrow corresponded with this diagnosis $(46, X X[6], 54$ $-X, 1 q+,+1 p-,+3,+5,+6 q-,+9,11 q+$, $-13,14 q+,+15,+16,+19,+20$ [4]). She was treated with intensive chemotherapy and autologous stem cell transplantation. Her father (II-3) had been diagnosed with $M M$ at 71 years of age after the discovery of a lytic lesion in the spine and an $\lg G$ paraproteinaemia. No cytogenetic analysis was performed. He was treated with chemotherapy and radiotherapy. Four years after the diagnosis he died from complications due to MM. The brother of the index patient (III-1) had been diagnosed with MM at 45 years of age. He had several lytic bone lesions in his spine and pelvis and an $\lg G$ paraproteinaemia

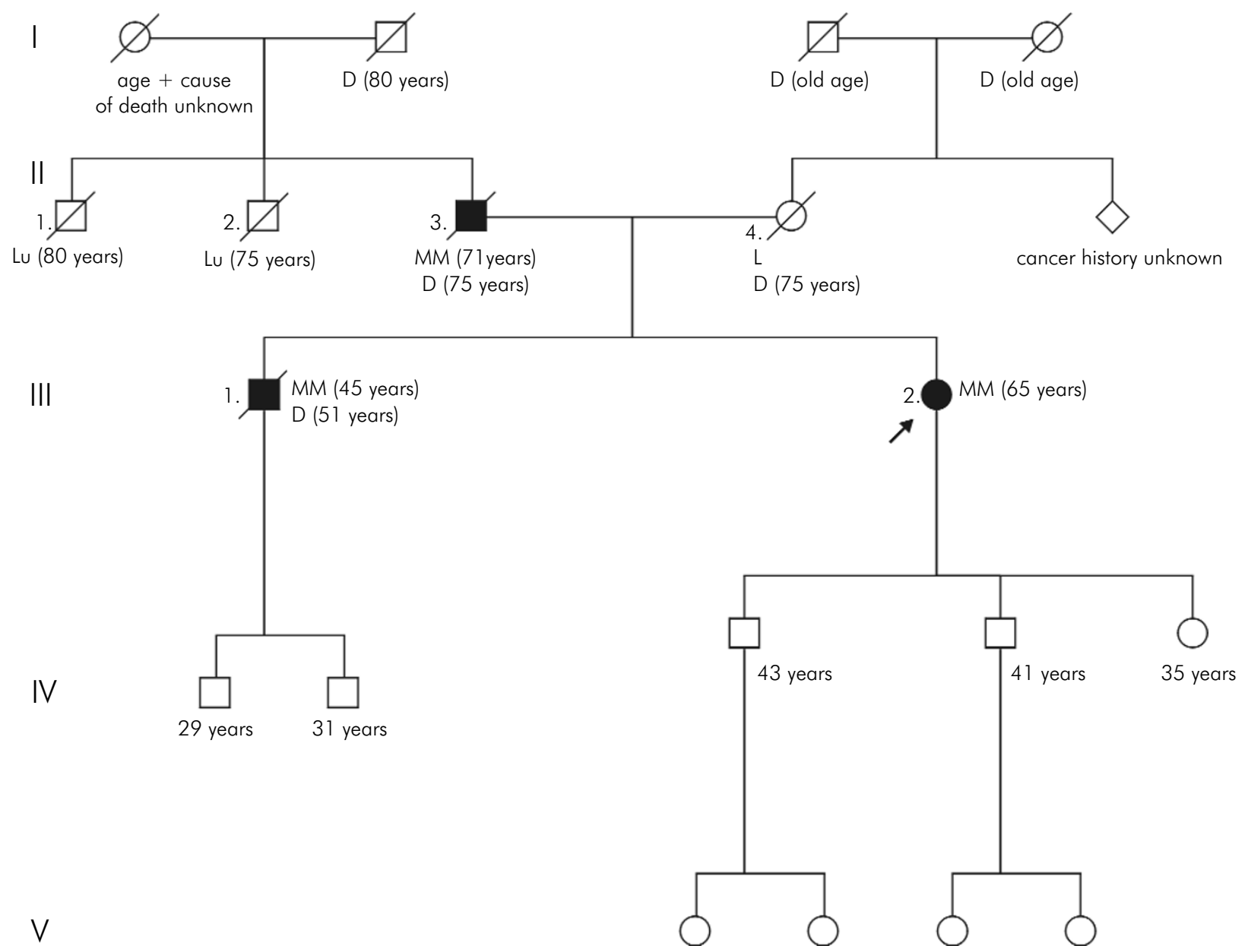

Fig. 1. Pedigree of family 1 with multiple myeloma. Diagnosis and age of diagnosis are given, as well as age of death. Solid blocks represent patients with multiple myeloma; open blocks represent patients without multiple myeloma. Diagonal lines indicate deceased individuals, and the arrow indicates the proband. The roman numbers in the left margin refer to the different generations. MM = multiple myeloma; $\mathrm{Lu}=$ lung cancer; $\mathrm{L}=$ leukaemia; $\mathrm{D}=$ deceased; number in parenthesis = age of onset, current age, or age of death 
of $140 \mathrm{~g} / \mathrm{l}$. Cytogenetic analysis of bone marrow showed no abnormalities (46,XY [10]). He was treated with intensive chemotherapy, radiotherapy and autologous stem cell transplantation. Six years after diagnosis he died of complications due to MM.

The mother of the index patient died of leukaemia (type unknown) at 75 years of age. No other haematological malignancies were reported in the family. Two of the patient's paternal uncles died of lung cancer at an old age (smoking habits unknown). There was no known shared or individual exposure to toxic chemicals or radiation in the patients.

Family 2 had two first-degree relatives affected by MM (Figure 2). The index patient is a 38-year old male who was diagnosed with MM after discovery of a lytic lesion in the hip. He had an $\lg G$ paraproteinaemia of 37 $\mathrm{g} / \mathrm{l}$ and six percent bone marrow plasma cells. Cytogenetic findings in bone marrow were normal $(46, X Y$ [10]). He underwent radiotherapy, intensive chemotherapy and stem cell transplantation.

His father was diagnosed with $M M$ at the age of 60 years after having a pathological fracture of the upper arm. He had an IgA-lambda paraproteinaemia of $14 \mathrm{~g} / \mathrm{l}$ and thirty percent bone marrow plasma cells. Cytogenetic findings in bone marrow corresponded with this diagnosis $(46, X Y[4], 58, X Y,+3,+5,+6$, $+(8 ; 22)(q 24 ; q 11),+9,+11,+15,+18,+19,+21$ [6]). He was treated with intensive chemotherapy and autologous stem cell transplantation.
The patterns of other types of cancer seen in these two families did not suggest any known hereditary cancer syndrome.

\section{Discussion}

Multiple myeloma is a relatively rare haematological malignancy of older persons. It has an unknown aetiology. It usually occurs incidentally within a family, but several familial cases have been reported in the literature [5-11]. The frequency of familial MM seems to be approximately 3.2 per 1000 cases of MM, leading to an occurrence of familial MM in approximately 1 per 10 million persons per year, making it a rare event [5].

The familial clustering of MM might be explained in several ways. Shared environmental factors have been implicated, for example radiation and pesticide exposure were reported to have led to a higher incidence of MM [12-13]. However, Alexander et al. concluded that no environmental risk factors could consistently be established when they compared multiple epidemiologic studies on environmental influences [14]. Chance alone might have caused the clustering within families; however, several families have shown an inheritance pattern that is very suggestive of an underlying genetic factor. A number of authors have proposed an autosomal dominant mode of inheritance, with reduced penetrance, to explain the occurrence of multiple cases of $M M$ within a family $[6-11,15]$.

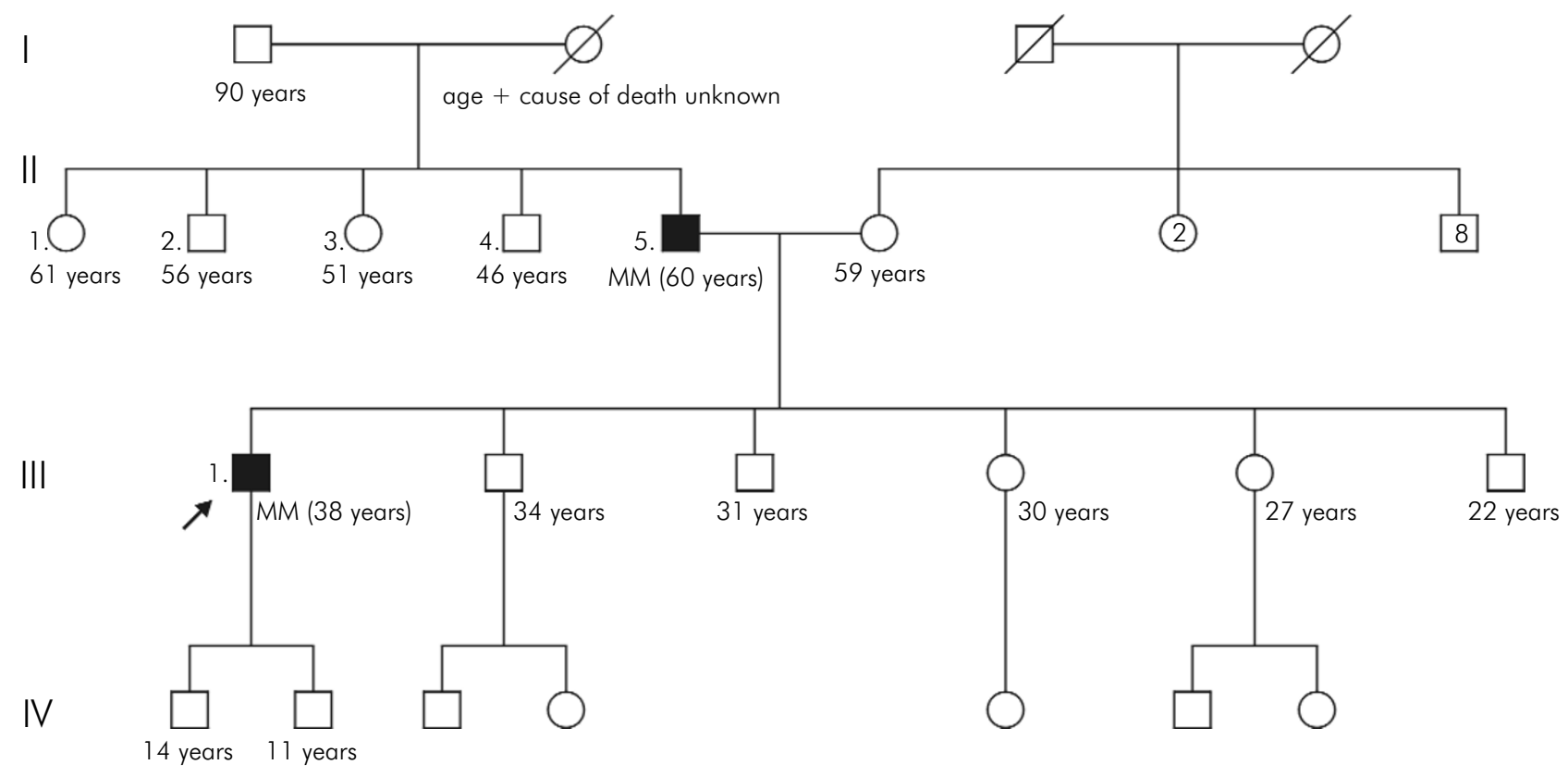

Fig. 2. Pedigree of family 2 with multiple myeloma (see Figure 1 for explanation of symbols) 
Engelhardt et al. postulated an autosomal recessive inheritance with low penetrance based on their findings of a high prevalence of B-cell diseases (MM or MGUS - monoclonal gammopathy of unknown origin) among siblings of MM patients. However, they only screened siblings and showed no data on paternal or offspring disease state [16].

Both our families would fit the hypothesis of an autosomal dominant pattern of inheritance, although environmental agents, low penetrance predisposing genes, or clustering by chance - or combinations of these - could be alternative explanations for the familial pattern. Unfortunately, because of the small number of informative meioses and the lack of DNA samples from some of the affected relatives, linkage analysis cannot be expected to solve the puzzle in these two families.

In the first family all three affected persons had an Ig $G$ paraproteinaemia, which might suggest a common genetic cause, but in the second family the type of monoclonal immunoglobulin differed between the two patients. A discrepancy in type of immunoglobulin produced within a family was also shown by Lynch et al. [6] in an MM/MGUS family and also by Ogmundsdottir [17].

Some studies suggest that germline gene mutations and polymorphisms may be associated with a risk of MM; however, results have not been consistent and no causative inherited genetic factor has been detected so far $[14,18]$.

It has been well established that $M M$ is characterised by extensive genomic abnormalities in tumour cells. The abnormalities consist of numerical and gross structural changes, deletions, duplications and translocations. These changes are also found in most patients with MGUS. Some of these somatic cytogenetic defects have major prognostic value [19-20]. Only two of the patients in our families showed characteristic complex chromosomal changes, refuting a relation between specific somatic chromosomal abnormalities and constitutional genetic factors. However, only conventional cytogenetic analysis was performed in our patients. In theory, small structural chromosomal changes (shared by relatives) could have been overlooked due to the limited spatial resolution of the technique.

\section{Family history and cancer risk}

Because no genetic factor or exact mode of inheritance has been determined for $M M$, the risk for healthy relatives of MM patients can only be estimated from large case-control or cohort studies. Altiery et al. quantified the familial risk of MM by studying 11,752 patients with MM. They observed 32 familial cases. These cases showed an SIR (standardised incidence ratio) of MM in offspring from an affected parent of 2.45. The SIR of MM in siblings of an affected person was 2.44. The risk was highest for daughters of an affected mother (SIR 5.49) [10]. Landgren et al. conducted a case-control study to evaluate the risk of developing MM for someone with first-degree relatives with MM. They found a significantly increased risk for MM in relatives of cases (RR (relative risk) 1.67). Among female relatives, risks were almost four times higher [1 1]. No explanation was found for this gender difference. Hemminki found an SIR of 4.25 for MM among offspring with a parent presenting with $M M$ [21]. Eriksson et al. calculated an RR of 5.64 for first-degree relatives of MM patients [22]. The Swedish national cancer registers were used for the above studies. Outcomes presumably vary because they used different cohorts and different statistical methods. Brown et al. found an almost four-fold excess risk for MM in a large case-control study in the US among subjects with a first-degree relative with MM [23].

From the Icelandic Cancer Registry, Ogmundsdottir et al. calculated a significantly increased risk of developing MM for first-degree relatives (RR 2.33), with the risk being largest for female relatives (RR 3.23) [17]. Overall, the risk of MM in first-degree relatives of cases with MM seems to be between two- and four-fold. Similar familial risks have been reported for cancers with a known, highly penetrant, predisposition gene, like breast cancer, colon cancer and ovarian cancer $[9,24]$.

To the best of our knowledge, there are no reports estimating the risk for subjects with more than one (firstor second-degree) relative suffering from $M M$, as seen in our families, but we presume the risks are higher for relatives of familial cases of MM than for those with only a single family member suffering from MM.

The risk of developing other types of cancer seems to be slightly higher for relatives of MM patients. An increased risk for other lymphoproliferative disorders in the relatives of MM patients has been shown, especially for CLL (chronic lymphatic leukaemia) and NHL (non-Hodgkin lymphoma) [9-1 1, 23]. Significant associations were found between $M M$ in a patient and rectal, stomach, cervical, prostate, bladder, endocrine gland and connective tissue malignancies in family members [10, 21-22].

It has been shown that familial cases of MM occur at an earlier age than sporadic cases, which is a hallmark of heritable cancer [9-10]. Anticipation, or the tendency in a multi-generational family for cancer 
to occur earlier in subsequent generations, was observed in some studies for MM [5, 7-10], but not in all [25]. In general, anticipation could be explained by ascertainment bias (due to screening of relatives or to them being more aware of the disease, thus leading to earlier discovery) or changes of incidence over time, but it might also point towards a genetic cause of the disease $[9,17]$. In both our families there seems to be anticipation. The cancer occurred on average 16 years earlier in the first family, and 23 years earlier in the second family.

\section{Screening of asymptomatic relatives in multiple myeloma families}

Patients from families like the ones reported here often have questions regarding the possibly hereditary nature of $M M$ in their family and ask if there is a need to screen healthy relatives. There are no primary preventive measures for $M M$ as yet [14] and no consensus guidelines for screening healthy relatives in MM families. The only recommendation we could find in the literature is from Hodgson et al., who suggested screening using blood and urine protein electrophoresis in first-degree relatives of familial MM patients and follow-up surveillance of individuals with MGUS, but they did not specify the frequency of screening or the preferred age for starting screening [26].

A population with a high risk of $M M$ in which screening is recommended is the group of patients with MGUS (monoclonal gammopathy of unknown origin). MGUS can be seen as a pre-malignant precursor of $M M$ and it is defined by the presence of a monoclonal protein, but the absence of a large amount of plasma cells in the bone marrow and end-organ damage characteristic for MM (renal insufficiency, lytic bone lesions, anaemia, hypercalcaemia) [9-14]. MGUS progresses into $M M$ in about $1 \%$ of cases per year and progression cannot be prevented [27-28]. Periodic screening is therefore advised for patients with MGUS in order to detect progression into MM and to prevent or delay serious complications such as renal failure or pathologic fractures. The quality of life of patients can be improved by averting these events and the cost of long-term dialysis or surgical intervention for skeletal complications would be reduced [27]. Since there are no known predictors to indicate which MGUS patients will progress to $M M$, screening is recommended for all patients. The periodic screening should include annual serum protein electrophoresis [29].

Detection of MGUS in the relatives of MM patients has been reported several times $[5-6,9,11]$. These cases were either found by collecting familial cases or by direct screening of relatives. Lynch et al. reported on a family with three siblings suffering from MM. After screening 19 relatives using electrophoresis of urine and blood, they detected two siblings with MGUS. These individuals were advised about long-term follow-up because of their increased risk for MM [9].

When considering the usefulness of a screening program the Wilson and Jungner criteria are often used [30]. These indicate that there should be an important health problem in order to start screening and that it should only be offered if there is a certain degree of increased risk. The previously calculated relative risks (RRs) do not translate into a high lifetime risk because of the low risk for $M M$ in the general population, and they therefore do not carry the same weight as similar RRs that have been calculated for relatives of patients with common types of cancer like colorectal cancer or breast cancer [24]. However, although familial MM is very rare, screening should be offered to selected families for whom the health problem is significant. The exact risk is unknown for relatives of familial MM cases. Calculations did not take more than one affected relative into account and may therefore have significantly underestimated the risk for relatives in the rare families with multiple cases of MM. Indeed, several observations have suggested the existence of hereditary $M M$, and germline gene mutations with significant penetrance may therefore underlie familial MM. In the absence of known risk estimates for the relatives in these families, we conclude for practical purposes that a significant risk cannot be ruled out at the present time and the notion of screening in these families cannot simply be dismissed.

According to the Wilson and Jungner criteria, there should be a suitable and acceptable diagnostic test for the disease and good treatment options. Screening for $M M$ can be done by blood and urine protein electrophoresis, which is minimally invasive and relatively easy to perform. On detecting a monoclonal protein, a distinction between MGUS or MM should be made by further study, possibly including a bone marrow biopsy. Treatment options for MM have improved over the last few years with respect to survival and quality of life $[4,31]$.

The goal of cancer screening in general is to detect a pre-malignant, treatable lesion (as in the case of screening for adenomatous colorectal polyps) or to detect a cancer in an early, treatable stage (as in screening for breast cancer), and early detection should positively influence the natural cause of the disease. Even though MM is an incurable disease in most cases and preventing the benign precursor MGUS from progressing is impossible, screening might nevertheless 
be beneficial because detection of MGUS or early-state MM is advantageous for morbidity and delays mortality from the disease [27]. The criteria further state that screening should be cost-effective and continuous. The screening test for MM is cheap, and early detection could prevent costly complications. Screenings can be repeated at a certain interval.

The disadvantages of screening for MM are the possible distress for relatives in whom MGUS or MM is detected, since MGUS and MM are not curable and progression from MGUS to MM can neither be predicted nor prevented. However, weighing the advantages and disadvantages for screening of healthy relatives in MM families, we conclude that screening might be beneficial for this distinct group. We suggest that screening should comprise annual protein electrophoresis of blood and urine and that it should be restricted to individuals with more than one first-degree affected relative or to those with one first-degree and at least one second-degree relative with $M M$. Since $M M$ is rarely diagnosed before age 40, we propose that relatives should be screened from this age. If families present with $\mathrm{MM}$ at a younger age, then screening could be started five years earlier than the youngest age at diagnosis (with the five years being an arbitrary period). Counselling the families on the limitations and possible advantages of screening should of course precede any screening. We suggest that this screening should be performed in a research setting where the outcome can be closely monitored to determine its clinical value and the psychological burden. If, in the future, germline gene mutations are identified as the cause of $M M$, more accurate risk estimates will become available. If lifetime risks for mutation carriers should indicate the need for screening, then presymptomatic DNA testing can help in targeting this surveillance.

In summary, families with multiple cases of MM are rare. Although risk estimates for asymptomatic relatives in these families are not yet available, a clinically significant risk of developing MM cannot be excluded. In a research setting, these families could be offered screening for $M M$.

\section{References}

1. Munshi NC, Tricot G, Barlogi B. Plasma Cell Neoplasms. In: DeVita Jr. VT, Hellman S, Rosenberg SA. Cancer: Principles and Practice of Oncology; 6th ed. Philadelphia, PA: Lippincott Williams \& Wilkins 2001: 2465-2499.

2. Selby P, Gore M. Myeloma and Other Plasma Cell Malignancies. In Peckham M, Pinedo H. Veronesi U. Oxford Textbook of Oncology. Vol. 2. Oxford, Oxford University Press 1995: 1852-1878.

3. Association of Comprehensive Cancer Centres, The Netherlands. 2003.

4. Dispenzieri A, Rajkumar SV, Gertz MA, Fonseca R, Lacy $M Q$, Bergsagel PL, Kyle RA, Greipp PR, Witzig TE, Reeder CB, Lust

\section{Screening for healthy relatives in familial multiple myeloma}

\section{Inclusion criteria}

Individuals with

- more than one first-degree relative diagnosed with multiple myeloma, or

- one first-degree and at least one second-degree relative with multiple myeloma

\section{Screening programme}

Annual protein electrophoresis to test immunoglobulins in blood and urine, starting at age 40 years. If families present with MM at a younger age, then screening could be started at say five years earlier than the youngest age at diagnosis in those families.

This screening should be performed in a research setting

JA, Russell SJ, Hayman SR, Roy V, Kumar S, Zeldenrust SR, Dalton RJ, Stewart AK. Treatment of newly diagnosed multiple myeloma based on Mayo Stratification of Myeloma and Risk-adapted Therapy (mSMART): consensus statement. Mayo Clin Proc 2007; 82: 323-34 1

5. Grosbois B, Jego P, Attal M, Payen C, Rapp MJ, Fuzibet JG, Maigre M, Bataille R. Familial multiple myeloma: report of fifteen families. Br J Haematol 1999; 105: 768-770.

6. Lynch HT, Sanger WG, Pirruccello S, Quinn-Laquer B, Weisenburger DD. Familial multiple myeloma: a family study and review of the literature. J Natl Cancer Inst 2001; 93: 1479-1483.

7. Sobol H, Vey N, Sauvan R, Philip N, Noguchi T, Eisinger F. Re: familial multiple myeloma: a family study and review of the literature. J Natl Cancer Inst 2002; 94: 461-462.

8. Segel GB, Lichtman MA. Familial (inherited) leukemia, lymphoma, and myeloma: an overview. Blood Cells Mol Dis 2004; 32: 246-261.

9. Lynch HT, Watson P, Tarantolo S, Wiernik PH, Quinn-Laquer B, Isgur Bergsagel K, Huiart L, Olopade OI, Sobol H, Sanger W, Hogg D, Weisenburger D. Phenotypic heterogeneity in multiple myeloma families. J Clin Oncol 2005; 23: 685-893.

10. Altieri A, Chen B, Bermejo JL, Castro F, Hemminki K. Familial risks and temporal incidence trends of multiple myeloma. Eur $\mathrm{J}$ Cancer 2006; 42: 1661-1670.

11. Landgren O, Linet MS, McMaster ML, Gridley G, Hemminki K, Goldin LR. Familial characteristics of autoimmune and hematologic disorders in 8,406 multiple myeloma patients: a population-based case-control study. Int J Cancer 2006; 118: 3095-3098.

12. Baris D, Silverman DT, Brown LM, Swanson GM, Hayes RB, Schwartz AG, Liff JM, Schoenberg JB, Pottern LM, Greenberg RS, Stewart PA. Occupation, pesticide exposure and risk of multiple myeloma. Scand J Work Environ Health 2004; 30: 215-222.

13. Lewis EB. Leukemia, multiple myeloma, and aplastic anemia in American radiologists. Science 1963; 142: 1492-1494.

14. Alexander DD, Mink PJ, Adami HO, Cole P, Mandel JS, Oken MM, Trichopoulos D. Multiple myeloma: A review of the epidemiologic literature. Int J Cancer 2007; 120 Suppl 12: 40-61.

15. Horwitz LJ, Levy RN, Rosner F. Multiple myeloma in three siblings. Arch Intern Med 1985; 145: 1449-1450.

16. Engelhardt $M$, Ihorst $G$, Behringer D, Finke J, Roth B. Incidence of monoclonal $\mathrm{B}$-cell disease in siblings of patients with multiple myeloma. Haematologica 2006; 91: 274-276.

17. Ogmundsdottir HM, Haraldsdottirm V, Johannesson GM, Olafsdottir G, Bjarnadottir K, Sigvaldason H, Tulinius H. 
Familiality of benign and malignant paraproteinemias. A population-based cancer-registry study of multiple myeloma families. Haematologica 2005; 90: 66-71.

18. Brown EE, Lan Q, Zheng T, Zhang Y, Wang SS, Hoar-Zahm S, Chanock SJ, Rothman N, Baris D. Common variants in genes that mediate immunity and risk of multiple myeloma. Int $\mathrm{J}$ Cancer 2007; 120: 2715-2722.

19. Higgins MJ, Fonseca R. Genetics of multiple myeloma. Best Pract Res Clin Haematol 2005; 18: 525-536.

20. Liebisch P, Dohner H. Cytogenetics and molecular cytogenetics in multiple myeloma. Eur J Cancer 2006; 42: 1520-1529.

21. Hemminki K. Re: familial multiple myeloma: a family study and review of the literature. J Natl Cancer Inst 2002; 94: 462-463.

22. Eriksson M, Hallberg B. Familial occurrence of hematologic malignancies and other diseases in multiple myeloma: a case-control study. Cancer Causes Control 1992; 3: 63-67.

23. Brown LM, Linet MS, Greenberg RS, Silverman DT, Hayes RB, Swanson GM, Schwartz AG, Schoenberg JB, Pottern LM, Fraumeni JF Jr. Multiple myeloma and family history of cancer among blacks and whites in the U.S. Cancer 1999; 85: 2385 . 2390.

24. Hemminki K, Eng C. Clinical genetic counselling for familial cancers requires reliable data on familial cancer risks and general action plans. J Med Genet 2004; 41: 801-807.

25. Daugherty SE, Pfeiffer RM, Mellemkjaer L, Hemminki K, Goldin LR. No evidence for anticipation in lymphoproliferative tumors in population-based samples. Cancer Epidemiol Biomarkers Prev 2005; 14: 1245-1250.

26. Hodgson SV, Foulkes WD, Charis E, Maher ER. A practical guide to human cancer genetics. Chapter 9. Blood and lymph. $3^{\text {rd }}$ ed. Cambridge, Cambridge University Press; 2007, 120-131.

27. Kyle RA, Therneau TM, Rajkumar SV, Offord JR, Larson DR, Plevak MF, Melton LJ. A long-term study of prognosis in monoclonal gammopathy of undetermined significance. N Engl J Med 2002; 346: 564-569.

28. Kyle RA, Therneau TM, Rajkumar SV, Larson DR, Plevak MF, Offord JR, Dispenzieri A, Katzmann JA, Melton LJ. Prevalence of monoclonal gammopathy of undetermined significance. N Engl J Med 2006; 54: 1362-1369.

29. Kyle RA, Rajkumar SV. Monoclonal gammopathy of undetermined significance. UpToDate 2006: http://www.utdol.com/utd/index.do.

30. Wilson JM, Jungner YG. Principles and practice of mass screening for disease. Bol Oficina Sanit Panam 1968; 65: 281 . 393.

31. Hussein MA. Multiple myeloma: most common end-organ damage and management. J Natl Compr Canc Netw 2007; 5: 170-178 